Technical Note

\title{
On Spatial Relations for Non-small Cells Lungs Cancer Interpretation
}

\author{
Nadeem Salamat ${ }^{1, *}$, V. B. Surya Prasath ${ }^{2,3,4}$ and Malik M. Saad Missen ${ }^{5}$ \\ 1 Department of Basic Sciences and Humanities, Khawaja Fareed University of Engineering and Information \\ Technology, Rahim Yar Khan, Pakistan; nadeem.salamat@kfueit.edu.pk \\ 2 Computational Imaging and Visualization Analysis Lab, Department of Computer Science, University of \\ Missouri-Columbia, Columbia MO 65211 USA; prasaths@missouri.edu \\ 3 Division of Biomedical Informatics, Cincinnati Children's Hospital Medical Center (CCHMC), Cincinnati, \\ OH 45229 USA \\ 4 Department of Biomedical Informatics, College of Medicine, University of Cincinnati, OH USA \\ 5 Department of Computer Science and Information Technology, The Islamia University of Bahawalpur, \\ Pakistan; saad.missen@iub.edu.pk \\ * Correspondence: nadeem.salamat@kfueit.edu.pk; Tel.: +92-332-686-8106
}

\begin{abstract}
Computers and artificial intelligence affect every field of life nowadays. In medical image interpretation automatic decision making using algorithms are used increasingly in every sub-field and computer aided diagnosis (CAD) is one of the main tools available to medical science today. CAD systems are used as an augmented option for both the medical practitioner and the patients, with image analysis and interpretation being of primary importance. In particular, spatial relations are used in knowledge representation, and these relations can be used for effective medical image interpretation. In this paper, we put forth an algorithm for defining non-small cells lungs cancer (NSCLC) stages in lungs images interpretation using topological spatial relations. We show an application case study in event motion predictions for lung cancer staging scoring - tumor, nodes and metastasis (TNM) - with combined topological and directional relations.
\end{abstract}

Keywords: computer aided diagnosis; topological relations; TNM system; non-small Cells; lung cancer

\section{Introduction}

Nowadays pulmonary diseases are increasing rapidly due to certain environmental conditions as well as man-made scenarios. One of the major among pulmonary diseases is the lung cancer that is growing rapidly with the passage of time and has drastic impacts and major causes of mortality in world [1]. According to a WHO study, while breast cancer causes the highest rate of death of women in Pakistan and second highest is the lung cancer whereas in men lung cancer is the highest rate of death. Taken as a whole, lungs cancer is the highest cause of deaths in Pakistan for both sexes. Lung cancer is one of the major respiratory system disease, which cause difficulty in breathing. From the advent of the twentieth century, lung cancer is proliferating rapidly. Lung cancer is now the second most common type of cancer worldwide. It is caused by various factors, and the most common cause of happening is due to smoking but an individual has chances of having cancer even if he or she does not indulge in smoking, with symptoms and causes varying accordingly. However, smoking is considered a major reason for lung cancer. The latest studies shows that ratio of smokers and non-smokers individuals having a chances of lungs cancer is 10:1. Cancer can grow in both or in one of the lungs. Cancerous cells can grow and replicate itself into other body parts as well. With the passage of time, tumor cells grow large in cells and create disturbance in lungs activities. There are various kinds of lung cancer. 
Primary lung cancer starts from lungs whereas a cancer that starts from neighboring body part and spread to the lungs after metasizes in body is termed as secondary lung cancer. When lungs going through discomfort, their ability to bloodstreams decline.

Different imaging techniques are used in clinical applications. The computers are introduced to these applications for automatic image interpretation and diseases diagnosis. Medical images acquisition used for these applications is two dimensional (2D), three (3D) dimensional, or higher dimensions. These techniques are magnetic resonance imaging (MRI), magnetoencephalography (MEG), 3D ultra sound imaging, computed tomography (CT), positron emission tomography (PET), single photon emission computed tomography (SPECT), functional MRI (fMRI), and difiusion weighted imaging (DWI). These detailed amount of information needs most modern techniques of medical image interpretation to provide better diagnosis and treatment options for clinical applications. It requires significant innovation in all aspects of image processing, such as image segmentation, image registration, visualization, compression and communication and interpretation.

Computer-aided diagnosis (CAD) provides a computerized diagnostic result and these results are used as a second opinion for the patients. The CAD assist radiologists in the diagnosis of diseases using the image analysis methods. Presently, CAD is used in the area of detection of breast cancer in mammograms, these algorithms are extendable for lungs images also. Currently, a large research effort has been devoted to the detection and classification of various lung diseases in thoracic computed tomography (CT) images. In CAD schemes in thoracic CT, nodule detection, distinction between benign and malignant nodules, and detection, characterization, and differential diagnosis of diffuse lung disease are included. The CAD schemes are used in clinical practice by providing radiologists with computer output as a second opinion. These schemes are further extendable for cancer staging and can be used to detect the development of changes in cancer stages. After the detection and labeling stage, the PET /CT images are segmented. Then the cancer staging is defined [2],[3], [4], these stages are performed manually by imaging experts. These stages depend upon the size, shape and location of the Tumor[1]. In this paper, we propose an automatic image interpretation algorithm to define the Lungs cancer stages.

The number of methods have been introduced to detect the lungs cancer in images in CT and PET images by method developed in $[5,6]$. The PET integrated images in the cancer grading (TNS) system are used to detect the tumors[4]. In the TNM lungs cancer staging $T$ is used as the size of the tumor, $\mathrm{N}$ is used as the Lymph Node status and $M$ is the abbreviation of distant Metastasis [7]. The eighth edition of the TNM staging system has been officially accepted by the Union for International Cancer Control (UICC) and the American Joint Committee on Cancer (AJCC). Although, the AJCC has delayed its implementation of the eighth edition of the staging system until 2018. In case of Non Small Cell Lung Cancer (NSCLC), the early staging of the diseases is crucial and it helps the Doctor to propose the proper medication and therapy. The medication for lungs cancer is prescribed according to its known type and its stage. Various treatments are available for different kinds of cancer.

The spatial relations are actively used in many fields of image analysis and knowledge representation. These class of relations is further studied in the multiple contexts of metric relations and directional relations. Topological relations is subclass of metric relations. The topological relations are developed in $[8,9]$ using the point set topology approach and region connected calculus (algebraic point of view) in [10-12]. These relations vary from region to region pairs [13,14]. [15]. The change in the spatial position of objects is exploited by the topological changes between objects in images[13,16-18] and reasoning about these changes are argued in [19]. The spatial relations are defined in image objects after the segmentation and labeling of objects. During the segmentation and labeling stage, the objects are segmented and labeled. Then spatial relations are defined between the required object pair. These spatial relations can also be used to define the cancer stages, such as, for defining the primary or secondary lungs cancer, Disjoint or the Tangent Proper Part or Non Tangent Proper Part topological relation can be used. Similarly $T$ staging also depend upon the position of a tumor in lungs, and its growth to the neighboring body parts, this is possible intuitively by determining the Externally 
Connected (EC) of the lung cancer and the neighboring body part such as spinal bone, heart, ribs etc. Some relations also need to determine the history of the spatial relations, such as Reach relation can be modeled as initially DC and then EC relation, such as modeled in [20]. In this paper, we develop the Lungs cancer staging in images based on the spatial relations between body parts.

This paper is arranged as follows. Some preliminary definitions, and lungs cancer stages are discussed in Section 2. Section 3 describes the intersection model of topological relations. Finally, Section 4 provides the conclusions.

\section{Lungs Cancer Staging of NSCLC}

\subsection{Preliminary Definitions}

Regular closed Sets: A set $A$ is called regular closed if $A=\overline{(\operatorname{Int}(A))}$ Diameter of a set: Diameter of a set $A$ is defined as

$$
D(A)=\max \left\{d\left(a_{1}, a_{2}\right) \mid a_{1}, a_{2} \in A\right\}
$$

Definition: Let $A$ and $B$ be pair of subsets of a topological space $X$. A topological relation between $A$ and $B$ is described by a triplet of values $(A, B, d)$, where $d$ is the topological relation.

\subsection{TNM System}

The current system for staging lung cancer is based on the Tumor - Node - Metastasis (TNM) classification This grading system involves the primary tumor $(\mathrm{T})$, the degree of lymhponode involvement $(\mathrm{N})$ and the presence of metastasis (M)[7]. The TNM classification system has been used as a standard by American joint committee on cancer to help doctors to identify the stags and types of cancer during interpretation. Due to advances in cancer categories every year, TNM system is upgraded regularly. Tumors, nodes and lymph nodes has been assigned a number or letter in TNM system. These letters are explained as

T: Refers to the tumor which is in its initial form or called primary tumor.

$\mathbf{N}$ : Term $N$ indicates whether nearby lymph nodes are effected by cancer or not.

M: Refers to metastasis. It can predict whether distant parts of body has been effected by cancer or not.

Following is the detail of each system.

\subsection{T-Staging}

This staging depends upon the size and location of the tumor in NSCLC in human lungs . Satellite nodules are also checked in this stage of evaluation. The $T$ category gives information about aspects of the original (primary) tumor, size, how deeply it has grown into the organ it started in, and whether it has grown into nearby tissues.

Numbers after the $T$ (such as $T_{1}, T_{2}, T_{3}$, and $T_{4}$ ) might describe the tumor size and or amount of spread into nearby structures. The higher the $T$ number, the larger the tumor and/or the more it has grown into nearby tissues.

\subsection{N Staging}

Another important screening is the occurrence of nodal, specifically for the ones who are suffering from mediastinal issues but do not found diseases of extra thoracic.The $\mathrm{N}$ category staging is used to check if cancer has lead to lymphatic nodes or not, this includes 
Table 1. T Staging in Lungs Cancer [21]

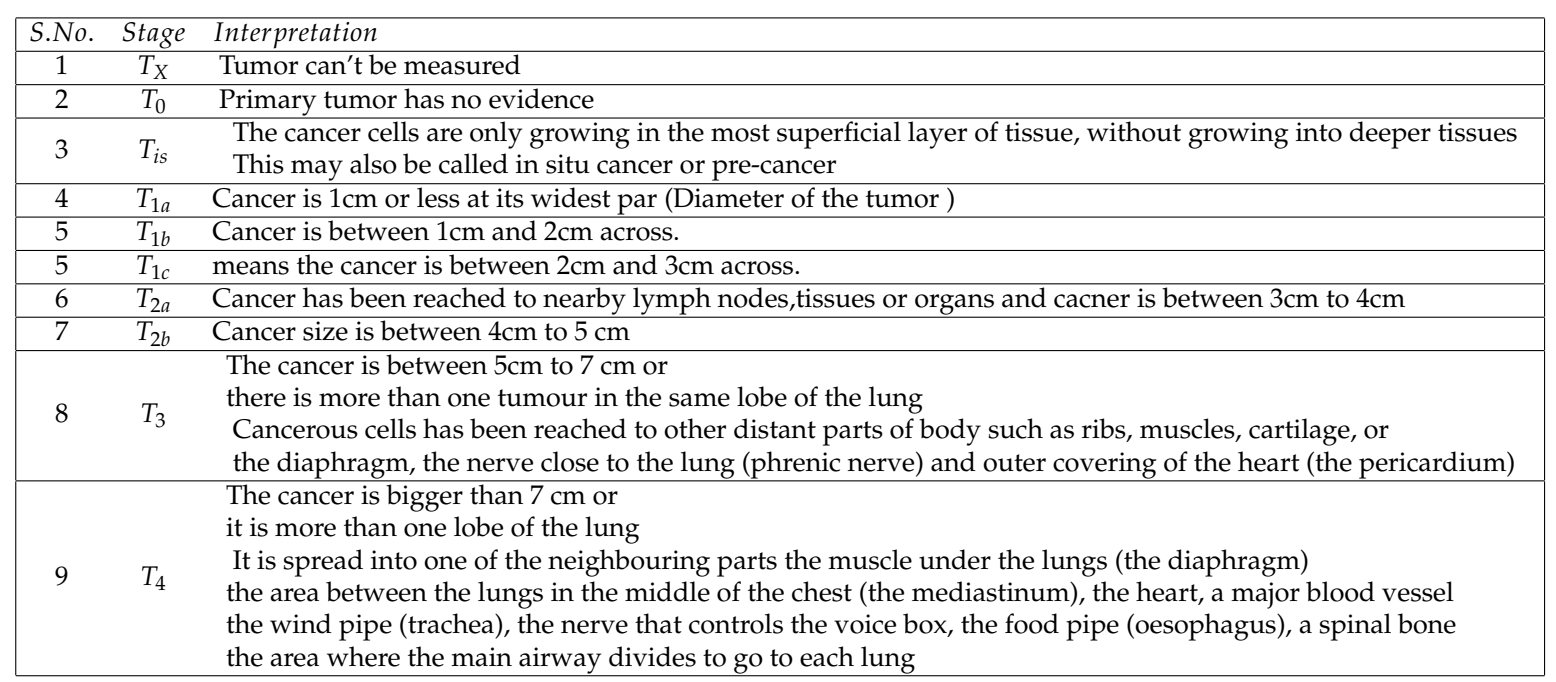

Table 2. N Staging in Lungs Cancer

\begin{tabular}{|ccl|}
\hline S.No. & Stage & Interpretation \\
\hline 1 & $N_{X}$ & Nearby lymph nodes can't be interpreted and evaluated. \\
\hline 2 & $N_{0}$ & No cancer in nearby lymph nodes. \\
\hline 4 & $N_{1}$ & $\begin{array}{r}\text { There are cancer cells in lymph nodes within the lung or } \\
\text { in lymph nodes in the area where the lungs join the airway (the hilum). }\end{array}$ \\
\hline 5 & $N_{2}$ & $\begin{array}{l}\text { in the centre of the chest (mediastinum) on the same side as the affected lung or } \\
\text { just under where the windpipe branches off to each lung }\end{array}$ \\
\hline 6 & $N_{3}$ & $\begin{array}{l}\text { means there is cancer in lymph nodes: } \\
\text { on the opposite side of the chest from the affected lung or } \\
\text { above the collar bone or at the top of the lung }\end{array}$ \\
\hline
\end{tabular}

\subsection{Staging}

The detection and identification of metastases occurred at distance spaces is another important task in lungs cancer staging. The $\mathrm{M}$ staging is described as

Table 3. M Staging in Lungs Cancer

\begin{tabular}{|c|c|c|}
\hline S.N & Stage & Interpretation \\
\hline 1 & $M_{0}$ & Nearby lymph nodes can't be interpreted and evaluated. \\
\hline 2 & M_1 $a$ & $\begin{array}{l}\text { means one or more of the following: } \\
\text { there is cancer in both lungs } \\
\text { there are areas of cancer around the heart or in the lining around the lung } \\
\text { there is fluid around the lung or heart that contains cancer cells, } \\
\text { this is called a malignant pleural effusion or a pericardial effusion }\end{array}$ \\
\hline 4 & M_1b & There is a single area of cancer outside the chest in an organ (such as the liver or brain) or a lymph node. \\
\hline 5 & M_1c & There is more than one area of cancer in one or several organs \\
\hline
\end{tabular}

\subsection{TNM Grading System}

The TNM classification of Non Small Cell Lungs Cancer is the classification system developed by the Pieter E. all in ([21]). This system is designed to help efficiently distinguish lung carcinomas from other lung lesions, as well as how cancer stages. The combination of the T, N, and M scores is then used to place a given lesion in one of four disease stages (I-IV).

As other factors helps in medications, same like is the case with grading. Grading can also be helpful in treatment decision of patients like other factors such as aging, cancer staging. These stages 
Table 4. Over all Staging in Lungs Cancer.

\begin{tabular}{|c|c|c|c|}
\hline S.No. & Stage & TNMCombination & Interpretation \\
\hline 1 & Stage $_{0}$ & & Nearby . \\
\hline 2 & Stage_1A & $\begin{array}{l}T_{1 a} N_{1} M_{0} \\
T_{1 b} N_{1} M_{0} \\
T_{2 c} N_{1} M_{0}\end{array}$ & The size of cancer is $3 \mathrm{~cm}$ or smaller \\
\hline 4 & Stage_IB & $T_{2 a} N_{0} M_{0}$ & $\begin{array}{l}\text { - Cancer is between } 3 \mathrm{~cm} \text { and } 4 \mathrm{~cm} \text {. It is growing into structures such as: } \\
\text { main bronchus, visceral pleura } \\
\text { - Cancer is blocking the airway, as a result the lung collapse partly or completely }\end{array}$ \\
\hline 5 & Stage_2A & $T_{2 b} N_{0} M_{0}$ & Cancer size is between $4 \mathrm{~cm}$ and $5 \mathrm{~cm}$, and no cancer cells in any lymph nodes. \\
\hline 6 & Stage_2B & $\begin{array}{l}T_{1 a}, N_{1}, M_{0} \\
T_{1 b}, N_{1}, M_{0} \\
T_{1 c}, N_{1}, M_{0} \\
T_{2 a} N_{1} M_{0} \\
T_{2 b} N_{1} M_{0} \\
T_{3} N_{0} M_{0}\end{array}$ & $\begin{array}{l}\text {-The tumor size is between } 3 \mathrm{~cm}-7 \mathrm{~cm} \text {, and it has spread to local lymph nodes; or } \\
\text { - The tumor is greater than } 7 \mathrm{~cm} \text { in size and has not spread to lymph nodes or } \\
\text { - Cancer is not in lymph node, but has spread to local areas such as, diaphragm, } \\
\text { phrenic nerve, mediastinal pleura and parietal pericardium. or } \\
\text { - the cancer is less than } 7 \mathrm{~cm} \text { but there are multiple tumours in the same lobe of the lung. }\end{array}$ \\
\hline 5 & Stage_3A & $\begin{array}{l}T_{1 a}, N_{2}, M_{0} \\
T_{1 b}, N_{2}, M_{0} \\
T_{1}, N_{2}, M_{0} \\
T_{2 a}, N_{2}, M_{0} \\
T_{2 b}, N_{2}, M_{0} \\
T_{3}, N_{1}, M_{0} \\
T_{4}, N_{0}, M_{0} \\
T_{4}, N_{1}, M_{0}\end{array}$ & $\begin{array}{l}\text { - Cancer is up to } 5 \mathrm{~cm} \text { in size and has spread to the lymph nodes in the centre of the chest on } \\
\text { the same side as the tumour or } \\
\text { - it is between } 5 \mathrm{~cm} \text { and } 7 \mathrm{~cm} \text { and multiple tumour in the same lobe of the lung or } \\
\text { - Cancer has spread into one or more of the following areas: the chest wall, } \\
\text { the phrenic nerve, mediastinal pleura and } \\
\text { parietal pericardium, lymph nodes in the lung or close to the lung or } \\
\text { - Cancer is larger than } 7 \mathrm{~cm} \text {. It hasn't spread into lymph nodes but has spread into } \\
\text { one or more of, diaphragm, mediastinum,the heart, a main blood vessel, } \\
\text { trachea, larynx, oesophagus, a spinal bone, the carina or } \\
\text {-Cancer in multiple lobes of the same lung and there might also be } \\
\text { cancer cells in lymph nodes close to the affected lung. }\end{array}$ \\
\hline 6 & Stage_3B & $\begin{array}{l}T_{1 a}, N_{3}, M_{0} \\
T_{1 b}, N_{3}, M_{0} \\
T_{1}, N_{3}, M_{0} \\
T_{2 a}, N_{3}, M_{0} \\
T_{2 b}, N_{3}, M_{0} \\
T_{3}, N_{2}, M_{0} \\
T_{4}, N_{2}, M_{0}\end{array}$ & $\begin{array}{l}\text {-Cancer is less than } 5 \mathrm{~cm} \text { and has spread into lymph nodes in one of these places: } \\
\text { the opposite side of the chest from the affected lung, the neck, above the collarbone, Or } \\
\text {-Cancer size is between } 5 \mathrm{~cm} \text { to } 7 \mathrm{~cm} \text { and has spread into lymph nodes in the centre of the chest. Or } \\
\text {-Cancer of any size, has spread into lymph nodes in the chest centre, and has spread into } \\
\text { any of the areas: chest wall, diaphragm, mediastinal pleura and parietal pericardium. Or } \\
\text { - Cancer has spread into the lymph nodes in the chest centre. The lung tumour is more than } 7 \mathrm{~cm} \\
\text { or it has spread into a major structure in your chest such as: the heart, trachea, } \\
\text { oesophagus, a main blood vessel }\end{array}$ \\
\hline 7 & Stage_3C & $\begin{array}{l}T_{3}, N_{3}, M_{0} \\
T_{4}, N_{3}, M_{0}\end{array}$ & $\begin{array}{l}\text { - Cancer is between } 5 \mathrm{~cm} \text { and } 7 \mathrm{~cm} \text { or has spread into one or more of the following: } \\
\text { phrenic nerve, parietal pericardium } \\
\text { - and it has spread into lymph nodes: } \\
\text { in the centre of the chest on the opposite side from the affected lung or at the top of } \\
\text { the lung on the same side or opposite side or above the collar bone } \\
\text { or there is more than one tumour in a different lobe of the same lung. Or } \\
\text { - Cancer is bigger than } 7 \mathrm{~cm} \text { or it has spread into one areas: (i) the diaphragm, } \\
\text { (ii) mediastinum, (iii) the heart, (iv) a major blood vessel (v), trachea, } \\
\text { (vi) the recurrent laryngeal nerve, oesophagus } \\
\text { a spinal bone, the area where the windpipe divides (the carina) } \\
\text { - It has spread into lymph nodes in chest center on the opposite side from the } \\
\text { affected lung or at the top of the lung on the same side or opposite side or above the collar bone }\end{array}$ \\
\hline 8 & Stage_4 & $\begin{array}{l}\text { AnyT, AnyN, M1a } \\
\text { AnyT, AnyN, M1b } \\
\text { AnyT, AnyN, M1c }\end{array}$ & $\begin{array}{l}\text { - Cancer in both lungs or the cancer is in the pleura or } \\
\text { pericardium or there is fluid around the lungs or the heart that contains cancer cells, Or } \\
\text { - Cancer has spread outside the chest to a lymph node or liver or bone. Or } \\
\text { - The cancer has spread to several areas in one or more organs }\end{array}$ \\
\hline
\end{tabular}

can be defined as the intersection of mathematical objects. In this system every object is defined as the closed set.This set is further divided into topological parts, like boundary, interior and exterior.At the next step, the topological relations are computed between the every object pair.

\section{Topological relations for lung cancer staging and proposed method}

The spatial relations between objects in images are used in many image analysis applications and medical image interpretation is one of them. [22]. In point set topological notation, these relations are defined as the intersection of two object as 9 intersections model [17]. In this model, topological primitives participate in defining the relations between parts, these parts are, closure, boundary, interior and exterior. For example, an object $A$ can be written into its body parts as $A^{c}$ (exterior), $\partial A$ (boundary), and $A^{o}$ interior of object $A$. Relation between two objects is developed by the void $\varnothing$ and non void intersection $\neg \varnothing$ entries of the matrix.

The matrix method represents the set of 9-intersections and each combination of 9 intersections describes a different topological relation, the 9- intersection can be employed to analyze whether or not two different configurations have the same topological relation [13,16-18]. Examples of topological invariants applicable to the 9-intersection are the content (i.e., Region of two body parts is empty or 
non empty) of a set, the dimension, the number of separations, and the sequence of disconnected intersections of different dimensions along the boundary. For the 9-intersection mode, the of the nine intersections was identified as a simple and most general topological invariant. It characterizes each of the nine intersections by a value $(\varnothing)$ or $(\neg \varnothing)$. For example, the 9-intersections based on empty/non-empty intersections for each configuration is

Disjoint(DC): In this situation, two objects are disjoint and topological parts of both the objects don't intersect each other. In matrix notation, this situation can be described as

$$
D C(A, B)=\begin{array}{|c|c|c|c|}
\hline \cap & B^{c} & \partial B & B^{o} \\
\hline A^{c} & \neg \varnothing & \neg \varnothing & \neg \varnothing \\
\hline \partial A & \neg \varnothing & \varnothing & \varnothing \\
\hline A^{o} & \neg \varnothing & \varnothing & \varnothing \\
\hline
\end{array}
$$

Externally Connected (EC): Where boundaries intersect but the interiors do not intersect

$$
E C(A, B)=\begin{array}{|c|c|c|c|}
\hline \cap & B^{c} & \partial B & B^{o} \\
\hline A^{c} & \neg \varnothing & \neg \varnothing & \neg \varnothing \\
\hline \partial A & \neg \varnothing & \neg \varnothing & \varnothing \\
\hline A^{o} & \neg \varnothing & \varnothing & \varnothing \\
\hline
\end{array}
$$

Partially Overlap (PO): In this case, the boundaries of both the objects intersect each other, similarly, interiors and exteriors of both objects intersect each other.

$$
P O(A, B)=\begin{array}{|c|c|c|c|}
\hline \cap & B^{c} & \partial B & B^{o} \\
\hline A^{c} & \neg \varnothing & \neg \varnothing & \neg \varnothing \\
\hline \partial A & \neg \varnothing & \neg \varnothing & \neg \varnothing \\
\hline A^{o} & \neg \varnothing & \neg \varnothing & \neg \varnothing \\
\hline
\end{array}
$$

Tangent Proper Part (TPP): In this situation, the reference region lies inside the targeted region and it touches the targeted boundary internally and 9 intersections are discussed as

$$
\operatorname{TPP}(A, B)=\begin{array}{|c|c|c|c|}
\hline \cap & B^{c} & \partial B & B^{o} \\
\hline A^{c} & \neg \varnothing & \neg \varnothing & \neg \varnothing \\
\hline \partial A & \varnothing & \neg \varnothing & \neg \varnothing \\
\hline A^{o} & \varnothing & \varnothing & \neg \varnothing \\
\hline
\end{array}
$$

Non Tangent Proper Part part Inverse (NTPP): In this situation, the 9 intersection matrix is represented as

$$
\operatorname{NTTP}(A, B)=\begin{array}{|c|c|c|c|}
\hline \cap & B^{c} & \partial B & B^{o} \\
\hline A^{c} & \neg \varnothing & \neg \varnothing & \neg \varnothing \\
\hline \partial A & \varnothing & \varnothing & \neg \varnothing \\
\hline A^{o} & \varnothing & \varnothing & \neg \varnothing \\
\hline
\end{array}
$$

Tangent Proper Part Inverse (TPPI): In this situation, boundary of the targeted object touches the boundary of reference object internally and 9-intersection is represented as bellow.

$$
\operatorname{TPPI}(A, B)=\begin{array}{|c|c|c|c|}
\hline \cap & B^{c} & \partial B & B^{o} \\
\hline A^{c} & \neg \varnothing & \varnothing & \varnothing \\
\hline \partial A & \neg \varnothing & \neg \varnothing & \varnothing \\
\hline A^{o} & \neg \varnothing & \neg \varnothing & \neg \varnothing \\
\hline
\end{array}
$$


NTPP I: In this situation, the reference object is smaller in size. It occupies space inside the argument object. The empty and non empty combination of nine intersections for the Non Tangent Proper Part Inverse (NTPPI) topological relation is described as

$$
\operatorname{NTTPI}(A, B)=\begin{array}{|c|c|c|c|}
\hline \cap & B^{c} & \partial B & B^{o} \\
\hline A^{c} & \neg \varnothing & \varnothing & \varnothing \\
\hline \partial A & \neg \varnothing & \varnothing & \varnothing \\
\hline A^{o} & \neg \varnothing & \neg \varnothing & \neg \varnothing \\
\hline
\end{array}
$$

Equal: The two objects are equal in size, then the topological relation between two objects is represented by the point set topology

$x=y$ for every point of the set and its matrix representation will be

$$
E Q(A, B)=\begin{array}{|c|c|c|c|}
\hline \cap & B^{c} & \partial B & B^{o} \\
\hline A^{c} & \neg \varnothing & \varnothing & \varnothing \\
\hline \partial A & \varnothing & \neg \varnothing & \varnothing \\
\hline A^{o} & \varnothing & \varnothing & \neg \varnothing \\
\hline
\end{array}
$$

\subsection{Segmentation and Labeling}

The spatial relations are defined between the segmented objects(body part) whose boundaries are well defined. These objects are considered as the regular sets where the boundary is the part of the object. The object recognition, segmentation and labeling of objects in an image is a separate problem.

During the object recognition stage, segmentation process of the lungs image, the body parts within the lungs or the close to lungs are segmented and then labeled as bellow and the tumor itself is labeled as object $A$. If there are multiple cancers regions are detected then they are labeled as $A_{i}, i=1,2, \ldots n$. Formally, for a given image, containing objects of interest and a set of labels corresponding to a set of models known to the system is considered as below

The topological relations used in the ontological of medical images can be renamed as Inside, this relation is developed by combining the TPP and NTPP defined in Eq (5 and Eq(6). If the out put of these relations is stored as 1 and zero, then Inside is defined as

$$
\operatorname{Inside}(A, B)=\max \{T P P(A, B), \operatorname{NTPP}(A, B)\}
$$

Similarly the second relation is defined topologically in $\mathrm{Eq}(3)$.

The NSLC staging can be developed using the Spatial relations between the cancerous cells of the body and different body parts. Once these body parts are segmented and labeled as described in above scheme, the spatial relations can describe the image contents and the cancer stage. The reach relation is defined in videos. In this case, each time analysis is considered as a snapshot, then if the topological relation between two consecutive test times is changed from disjoint to the externally connected. The concatenation of these two spatial relations is marked as the reach relation.

\subsection{Primary and Secondary Cancer Detection}

Once the objects are detected and labeled, the spatial relation disjoint as described in $\operatorname{Eq}(2)$ is computed between the objects $A$ and the lungs. If this relation holds, then the cancer type is the secondary. IF the output of this relation is zero, or the partially overlap relationship holds between the Lungs $\left(O_{19}\right)$ and the cancerous object $A$. In such a case, the cancer type will be based on the origin place of the cancer object.

Similarly a cancer is termed as the primary cancer if computed by the equations $(\mathrm{Eq}(5)$ and $\mathrm{Eq}(6)$ holds. Once, it is decided that the cancer is primary, then its stages are determined through the following process. 
Table 5. Objects Labeling at the segmentation stage

\begin{tabular}{|c|l|l|}
\hline S.No. & Label & Body Part \\
\hline 1 & $O_{1 L u}$ & upper lobe Left Lungs \\
\hline 2 & $O_{1 L l}$ & lower lobe of Left Lungs \\
\hline 2 & $O_{1 R u}$ & upper lobe of Right Lungs \\
\hline 2 & $O_{1 R m}$ & Middle lobe of Right Lungs \\
\hline 2 & $O_{1 R l}$ & Lower lobe of Right Lungs \\
\hline 3 & $O_{2 L}$ & Left Lungs airway (Main Bronchus ) \\
\hline 4 & $O_{2 R}$ & Right Lungs airway (Main Bronchus ) \\
\hline 5 & $O_{3 L}$ & Membrane covering the Left lungs ( Visceral Pleura ) \\
\hline 6 & $O_{3 R}$ & Membrane covering the Right lungs ( Visceral Pleura ) \\
\hline 7 & $O_{4}$ & Lymph nodes \\
\hline 8 & $O_{5}$ & Chest wall (Diaphragm ) \\
\hline 9 & $O_{6}$ & Phrenic nerve \\
\hline 10 & $O_{7}$ & Heart \\
\hline 11 & $O_{8}$ & Outer covering of the heart (Pericardium ) \\
\hline 12 & $O_{9}$ & Muscle under the Lungs \\
\hline 13 & $O_{10}$ & Central area of chest ( mediastinum) \\
\hline 14 & $O_{11}$ & Main blood vessel \\
\hline 15 & $O_{12}$ & Wind pipe(Trachea ) \\
\hline 16 & $O_{13}$ & Voice box (Larynx) \\
\hline 17 & $O_{14}$ & Food pipe ( Oesophagus) \\
\hline 18 & $O_{15}$ & Spinal bone ( carina ) \\
\hline 19 & $O_{16}$ & Neck (above the collarbone) \\
\hline 20 & $O_{17}$ & Liver \\
\hline 21 & $O_{18}$ & Ribs \\
\hline 22 & $O_{19}$ & Cartilage \\
\hline 24 & $O_{20}$ & the area where the windpipe divides (the carina) \\
\hline & & \\
\hline
\end{tabular}

\section{3. $T$ stages}

Once the cancer is decided as the primary cancer in lungs then at next stage, the TNM cancer stages are interpreted in terms of spatial relations and size of the tumor. The size of the tumor is measured as the object recognition stage of the system ends up.

The $T$ staging in cancer is based on two criteria, which are

- Size of tumor, this is determined by the equation (1)

- The intersection of the cancer and the different parts of the lungs.

The higher order $T$ staging is defined as the intersection of the cancer and the neighboring body parts of the Lungs. In this table the $\vee$ is used to represent the or (union ) and the $\wedge$ represents the and (intersection) operator. In detail, these are defined as

\section{4. $N$ Stages}

In the $N$ staging, the spatial relation partially overlap between the cancer image in the lungs and the body parts is defined as Eq.(4). For the $N_{x}$ and $N_{0}$ stages there is empty intersection of the cancerous part and the lymph nodes

\section{5. $M$ stages}

Grading schemas also depends upon cancer type, some cancer types doesn't show compatibility with such grading system, in this case following terminology is used. 
Table 6. T Staging in Lungs Cancer in Terms of spatial relations Between Body Parts

\begin{tabular}{|c|c|c|}
\hline S.No. & Stage & Interpretation \\
\hline 1 & $T_{X}$ & Tumor can't be measured \\
\hline 2 & $T_{0}$ & Primary tumor has no evidence \\
\hline 3 & $T_{\text {is }}$ & $\begin{array}{l}\text { The cancer cells are only growing in the most superficial layer of tissue, without growing into deeper tissues } \\
\text { This may also be called in situ cancer or pre-cancer }\end{array}$ \\
\hline 4 & $T_{1 a}$ & $D<1$ \\
\hline 5 & $T_{1 b}$ & $1 \mathrm{~cm}<D<2 \mathrm{~cm}$ \\
\hline 5 & $T_{1 c}$ & $2 \mathrm{~cm}<D<3 \mathrm{~cm}$ \\
\hline 6 & $T_{2 a}$ & {$\left[E C\left(A, O_{2 R}\right) \vee E C\left(A, O_{2 L}\right) \vee E C\left(A, O_{3 R}\right) \vee E C\left(A, O_{3 L}\right) \vee E C\left(A, O_{4}\right)\right]$ and $3 c m<D<4 c m$} \\
\hline 7 & $T_{2 b}$ & $4 \mathrm{~cm}<D<5 \mathrm{~cm}$ \\
\hline 8 & $T_{3}$ & $\begin{array}{l}5 \mathrm{~cm}<D<7 \mathrm{cmor}\left[\operatorname{Inside}\left(A_{1}, O_{1 L u}\right), \operatorname{Inside}\left(A_{2}, O_{1 L u}\right), \ldots,\right] \vee\left[\operatorname{Inside}\left(A_{1}, O_{1 L l}\right),\right. \\
\left.\text { Inside }\left(A_{2}, O_{1 L l}\right) \ldots,\right] \vee\left[\operatorname{Inside}\left(A_{1}, O_{1 R u}\right), \operatorname{Inside}\left(A_{2}, O_{1 R u}\right), \ldots,\right] \vee\left[\operatorname{Inside}\left(A_{1}, O_{1 R m}\right),\right. \\
\left.\text { Inside }\left(A_{2}, O_{1 R m}\right) \ldots,\right] \vee\left[\operatorname{Inside}\left(A_{1}, O_{1 R l}\right), \operatorname{Inside}\left(A_{2}, O_{1 R l}\right), \ldots,\right] \text { Or, } \\
\operatorname{EC}\left(A, O_{5}\right) \vee E C\left(A, O_{6}\right) \vee E C\left(A, O_{8}\right), \vee E C\left(A, O_{9}\right) \vee E c\left(A, O_{18}\right) \vee E C\left(A, O_{19}\right)\end{array}$ \\
\hline 9 & $T_{4}$ & $\begin{array}{l}D>7 c m \text { Or }\left[P O\left(A, O_{1 L u} \wedge O_{1 L l}\right)\right] \vee\left[P O\left(A, O_{1 R u} \wedge O_{1 R m}\right)\right]\left[P O\left(A, O_{1 R m} \wedge O_{1 R l}\right)\right] \text { Or } \\
P O\left(A, O_{5}\right) \vee P O\left(A, O_{7}\right) \vee P O\left(A, O_{10}\right) \vee P O\left(A, O_{11}\right) \vee \\
\left.\left.P O\left(A, O_{12}\right), P O\left(A, O_{13}\right) \vee P O\left(A, O_{14}\right) \vee P O\left(A, O_{15}\right) \vee P O\left(A, O_{20}\right)\right)\right]\end{array}$ \\
\hline
\end{tabular}

Table 7. N Staging in Lungs Cancer in Terms of spatial relations Between Body Parts

\begin{tabular}{|ccl|}
\hline S.No. & Stage & Interpretation \\
\hline 1 & $N_{X}$ & Nearby lymph nodes can't be interpreted and evaluated. \\
\hline 2 & $N_{0}$ & No cancer in nearby lymph nodes. \\
\hline 4 & $N_{1}$ & $P O\left(A, O_{4}\right)$ \\
\hline 5 & $N_{2}$ & $P O\left(A, O_{10}\right) \vee P O\left(A, O_{2 L}\right) \vee P O\left(A, O_{2 R}\right)$ \\
\hline 6 & $N_{3}$ & $P O\left(A, O_{4}\right) \vee P O\left(A, O_{16}\right) \vee P O\left(A, O_{12}\right)$ \\
\hline
\end{tabular}

\subsection{TNM staging}

These stages are defined as a combination of $T, N$ and $M$ stages. The $T$ stages defined in table (6) and $N$ stages are defined in table (7) and $M$ stages are defined in table (8). The TNM stages with the spatial relations are combined as described in table 4.

\subsection{Examples}

These spatial relations can be used to determine the cancer stage as the following example shows. After the detection stage, the size is measured by the governing equation (1) and suppose $D=1.5$. This provides an information that tumor size is $3 \leq D \leq 5$. The intersection of the object $A_{i}$ is taken with the lungs parts. Suppose the Inide $\left(A, O_{1 l u}\right) \neq 0$, this shows that the cancer is primary type and its location is in the upper lobe. Its $T$ stages is determined as $T_{1 b}$. If no cancer part is detected in the nearby lymph nodes, i.e. $P O\left(A, O_{i}\right) \neq 0 i=4,10,12,16,2 l, 2 R$. This will result as the $N$ stage is $N_{1}$. If the $M$ stage is also $M_{0}$, then the overall stage is $T_{1 b} N_{1} M_{0}$ which is Stage - IA. Further, the spatial relations can also be used to determine the growth or control over the cancer by comparing the current state of the cancer with the previous history of the patient. As in previous part of the example, the states may change to $T_{2 a}$, or $T_{2 b}$ depending on growth size. The $\mathrm{N}$ stage can change from $N_{1}$ to $N_{2}$ depending on the growth of tumor and it may reduce size depending on the control of the tumor. $\mathrm{M}$ stage can be changed from $M_{0}$ to $M_{1}$. In this way new stage could be Stage - IA, Stage - IB or stage Stage - IIA.

\section{Conclusions}

Spatial relations are increasingly used in many fields of knowledge representation and image interpretation. These relations are also used for interpretation of medical images. The paper is concerned with the application of topological spatial relations for defining the TNM staging the NSCLC. In this paper, an applicable model is proposed for the lungs image interpretation. This can be 
Table 8. M Staging in Lungs Cancer in Terms of spatial relations Between Body Parts

\begin{tabular}{|cll|}
\hline S.No. & Stage & Interpretation \\
\hline 1 & $M_{0}$ & Nearby lymph nodes can't be interpreted and evaluated. \\
\hline 2 & $M_{-} 1 a$ & $\begin{array}{l}\text { Inside }\left(A_{1}, O_{1 L}\right) \wedge \text { Inside }\left(A_{2}, O_{1 R}\right) \text { or Inside }\left(A_{2}, O_{1 L}\right) \wedge \operatorname{Inside}\left(A_{1}, O_{1 R}\right) \\
P O\left(A, O_{8}\right), P O\left(A, O_{3 R}\right), P O\left(A, O_{3 L}\right)\end{array}$ \\
\hline 4 & $M_{-} 1 b$ & $P O\left(A, O_{17}\right) \operatorname{or} P O\left(A, O_{4}\right)$ \\
\hline 5 & $M \_1 c$ & There are more that one cancers in multiple body parts \\
\hline
\end{tabular}

used as the second opinion of practitioners in the medical field. This approach will be extended to the interpretation of other types of cancer in images.

Author Contributions: N.S and V.B.S.P. conceived and designed the spatial relations model for lung cancer staging; N.S, V.B.S.P. and M.M.S.M. discussed the model and wrote the paper.

Conflicts of Interest: The authors declare no conflict of interest.

1. Hochhegger, B.; Alves, G.R.T.; Irion, K.L.; Fritscher, C.C.; Fritscher, L.G.; Concatto, N.H.; Marchiori, E. $\mathrm{PET} / \mathrm{CT}$ imaging in lung cancer: indications and findings. Jornal brasileiro de pneumologia : publicacao oficial da Sociedade Brasileira de Pneumologia e Tisilogia, 2015.

2. Rankin, S. PET/CT for staging and monitoring non small cell lung cancer. Cancer imaging : the official publication of the International Cancer Imaging Society 2008, 8 Spec No A, 27-31.

3. De Wever, W.; Ceyssens, S.; Mortelmans, L.; Stroobants, S.; Marchal, G.; Bogaert, J.and Verschakelen, J.A. Additional value of PET-CT in the staging of lung cancer: comparison with $\mathrm{CT}$ alone, PET alone and visual correlation of PET and CT. European Radiology 2007, 17, 23-32.

4. El-Hariri, M.A.; Gouhar, G.K.; Refat, A.M. Integrated PET/CT in the preoperative staging of lung cancer: A prospective comparison of $\mathrm{CT},\{\mathrm{PET}\}$ and integrated PET/CT. The Egyptian Journal of Radiology and Nuclear Medicine 2012, 43, 613 - 621.

5. Valente, I.R.S.; Cortez, P.C.; Neto, E.C.; Soares, J.M.; de Albuquerque, V.H.C.; Tavares, J.M.R. Automatic 3D pulmonary nodule detection in $\{\mathrm{CT}\}$ images: A survey. Computer Methods and Programs in Biomedicine 2016, $124,91-107$.

6. Kecheril, S.S.; Venkataraman, D.; Suganthi, J.; Sujathan, K. Automated lung cancer detection by the analysis of glandular cells in sputum cytology images using scale space features. Signal, Image and Video Processing 2015, 9, 851-863.

7. Giron, J.; Lacout, A.; Marcy, P.Y. Dealing with Lung Cancer $\{\mathrm{TNM}\}$ Classification. Journal of Thoracic Oncology 2016, 11, e77 - e78.

8. Egenhofer, M.J. A Formal Definition of Binary Topological Relationships. FODO, 1989, pp. 457-472.

9. Egenhofer, M.J.; Franzosa, R.D. Point Set Topological Relations. International Journal of Geographical Information Systems 1991, 5(2), 161-174.

10. Clementini, E.; Felice, P.D. Approximate Topological Relations. International Journal of Approximate Reasoning 1997, 16, $173-204$.

11. Cohn, A.G.; Bennett, B.; G, A.; Gooday, J.; Nicholas.; Gotts, N.M. Qualitative Spatial Representation and Reasoning with the Region Connection Calculus. Proceedings of the DIMACS International Workshop on Graph Drawing, 1994. Lecture Notes in Computer Science, 1997, pp. 89-4.

12. Salamat, N.; Zahzah, E. 2D Fuzzy Spatial Relations: New Way of Computing and Representation. Advances in Fuzzy Systems 2012, 2012, Article ID 167939, 15 pages.

13. Egenhofer, M.J.; Herring, J.R. Categorizing Binary Topological Relations Between Regions, Lines and Points in Geographic Databases. Departement of Survey Engineering, University of Maine. Technical report, University of Maine, 1994.

14. Billen, R.; Kurata, Y. Refining Topological Relations Between Regions Considering Their Shapes. Proceedings of the 5th International Conference on Geographic Information Science; Springer-Verlag: Berlin, Heidelberg, 2008; GIScience '08, pp. 20-37. 
15. Zlatanova, S. On 3D Topological Relationships. Int. Workshop on Database and Expert System Applications. IEEE Computer Society Press, 2000, pp. 913-919.

16. Delafontaine, M.; de Weghe, N.V.; Bogaert, P.; Maeyer, P.D. Qualitative Relations Between Moving Objects in a Network Changing Its Topological Relations. Inf. Sci. 2008, 178, 1997-2006.

17. Egenhofer, M.; Mark, D. Modeling Conceptual Neighborhoods of Topological Line-Region Relations. International Journal of Geographical Information Systems 1995, 9, 555-565.

18. Egenhofer, M.J.; Al-Taha, K.K. Reasoning about Gradual Changes of Topological Relationships. Proceedings of the International Conference On GIS - From Space to Territory; Springer-Verlag: London, UK, 1992; pp. 196-219.

19. Cohn, A.G.; Hazarika, S.M. Qualitative Spatial Representation and Reasoning: An Overview. Fundamenta Informaticae 2001, 46, 1-29.

20. Salamat, N.; Zahzah, E. Combined Topological and Directional Relations Based Motion Event Predictions. Pattern Recognition and Machine Intelligence - 4th International Conference, PReMI 2011, Moscow, Russia, June 27 - July 1, 2011. Proceedings, 2011, pp. 180-185.

21. Goldstraw, P.; Crowley, J.; Chansky, K.; Giroux, D.J.; Groome, P.A.; Rami-Porta, R.; Postmus, P.E.; Rusch, V.; Sobin, L. The IASLC Lung Cancer Staging Project: Proposals for the Revision of the TNM Stage Groupings in the Forthcoming (Seventh) Edition of the TNM Classification of Malignant Tumours. Journal of Thoracic Oncology 2007, pp. 706- 714 .

22. Hudelot, C.; Atif, J.; Bloch, I. Fuzzy Spatial Relation Ontology for Image Interpretation. Fuzzy Sets Syst. 2008, 159, 1929-1951. 